\title{
Mobile Augmented Reality Application Supporting Building Facades Visualization
}

\author{
Michał Bednarczyk and Tomasz Templin \\ University of Warmia and Mazury in Olsztyn, Faculty of Geoengineering, \\ Institute of Geodesy and Civil Engineering
}

\begin{abstract}
The use of mobile devices such as smartphones still increases over last years. Smartphone hardware capabilities are comparable with average personal computers. Thanks to the increasing computing power, their application is focused on more and more sophisticated and complex tasks. These includes among others augmented reality (AR) applications, which support precise GNSS localization and use signals from such devices like accelerometer or gyrocsope. They are able to create interactive, immersive real-time visualisation of any kind. The article presents a prototype Mobile Augmented Reality (MAR) Application for visualization of the building facades. It allows to support the original look of the building with the additional information from database. Software of this kind, can be used for overviewing, inspecting or work planning carried out on the facade of the building. As a part of the test, a prototype of the MAR application for Android has been prepared. Rest of the solution components have been presented in form of a concept, implementation of which is planned in near future. Basic technical assumptions are already developed, but details may change. The paper mentions also some important AR problems, especially positioning in urban environment and accurate registration of digital objects in the real world.
\end{abstract}

Keywords: Mobile Application, Augmented Reality, Technical Documentation of the Building, Spatial Information System of the Building.

\section{Introduction}

The management of building maintenance is an important topic around the world, especially in recent decades, with the incorporation of tools to automate the tracking information and potential follow-up. From the social point of view it is illogical to leave the building stock without control. (Serrat, Gibert 2011) This is the reason why many companies and institutions invest in modern IT solutions to improve the process of ongoing buildings inspection. Data collected in this process are primarily: field data, cartographic data, cadastral data as well as plot building/facade data, architectural characteristics, existing elements and materials and the state of damages at the time of inspection. (Serrat, et al. 2016). Data collected reliably and systematically play a special role in monitoring and analyzing the degradation of building facades. In this way, the monitoring and maintenance of valuable historic buildings and the planning of ongoing renovations are significantly supported. (Gibert et al. 2014). The data analysis process is automated using various IT tools, including GIS systems, which are widely known and used in many fields. They allow to combine and process spatial data and non spatial data from many sources at the same time. This gives an ability to perform analysis, plan and undertake decisions in real world.

As observed in past years, there's been a new technology developed, which is called Augmented Reality (AR). It gives the ability to present data in a new way, not available before. AR means any system that "augments", or overlays, the real world with digital 
information that seems to co-exist with the real world. (Azuma 1997) Over the past few decades, this once theoretical field has matured into a mass medium (Ahonen 2012) with applications spanning countless industries. Speaking of AR, which is used in mobile devices, many authors (Höllerer, Feiner 2004; Henrysson 2007) use the term MAR (Mobile Augmented Reality), to clarify the scope of its application. AR technology, can be understood as a special video effect or real time digital image editing. It is similar to the techniques used in film editing or television. In this way, the illusion of "extending" or "augmenting" the observed reality with additional information and images can be obtained.

Generally, the combination of digital image with the real view is obtained by the use of the digital camera and the sensors like GPS/GNSS receiver, inclinometer, magnetometer, accelerometer, compass and other, which provide information about the location and orientation of the mobile device. Siltanen (Siltanen 2012) writes that AR technology connects two fields like:

- computer graphics needed for photorealistic rendering and interactive animations,

- computer vision used for marker and feature detection and tracking, motion detection and tracking, image analysis, gesture recognition and the construction of controlled environments containing a number of different sensors.

- Taking into account other technologies needed to develop a working AR application, also should be mentioned such fields as:
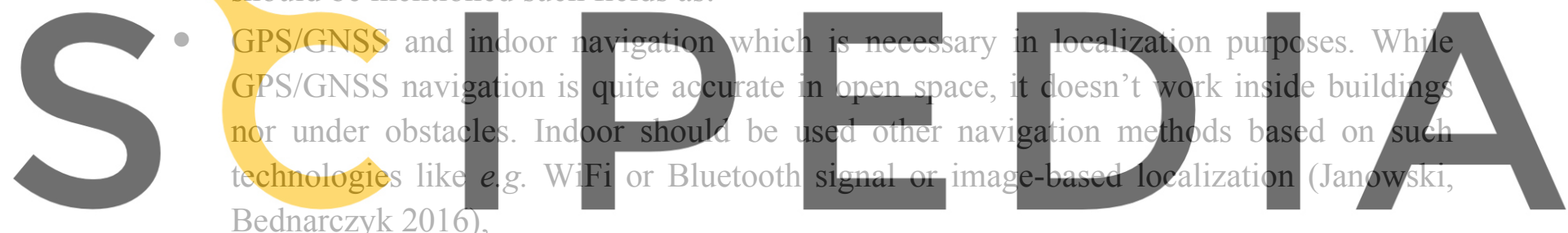

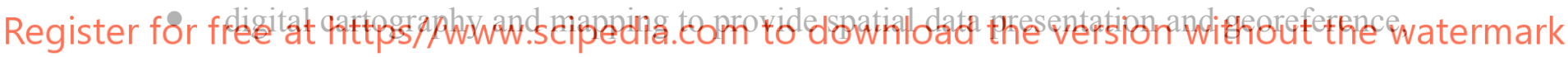

- WWW applications technologies, Service Oriented Architecture (SOA), Database

Management Systems (DBMS) and other technologies for data storage, sharing and communication.

AR starts to be widely-used and ideas for its usage can be multiplied almost indefinitely. The most obvious and popular seems to be the usage in tourism, where tourist attractions, can be observed directly on the smartphone screen, giving additional information during the tour (Kounavis et al. 2012). Application of this technology can be found in many other fields, where presentation of spatial data is needed. For example in architecture AR is used to present the three-dimensional models of buildings, housing estates and cities, visualization of the construction details, including installation and equipment or interior designing and decoration (Broschart et al. 2013; Xiangyu 2009; VTT 2013). AR is also used in spatial designing and planning for presentation of three-dimensional scale models and visualizations of projects in a real environment. Augmented reality maps, the content of which is expanded with additional information, descriptions, graphics or 3D objects, also seems to be an interesting spatial data presentation form (Moloney, Bharat 2011).

Observing such a variety of applications, the hypothesis can be formulated, that AR technology can be helpful in the building inspection process. Generally, this process is based on a comparison of the current state of the object with the state from previous years. On this 
basis, conclusions may be formulated regarding further actions. An important element here is the visualization of the current and historical state in the context of the building being under study.

\section{How does Augmented Reality Work}

AR Glossary, document published by the AR Community, mentions that augmentation means a relationship between the real world and a digital asset (AR Community 2017). It can be said that AR mixes virtual objects like graphics, sounds, videos,3D models etc. with the view of actual world. The effect of an augmentation is a composed scene. Augmentation may be formalized through an authoring and publishing process where the relationship between real and virtual is defined and made discoverable.

There are two primary techniques for image generation which lead to obtain the AR effect (Lechner 2015; AR Community 2017), they are:

1. Geospatial based Augmented Reality.

2. Computer-vision based Augmented Reality.

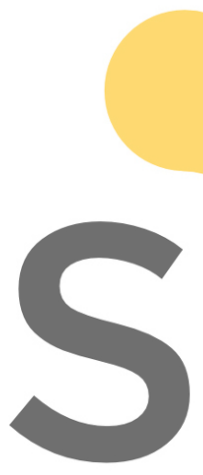

(1)
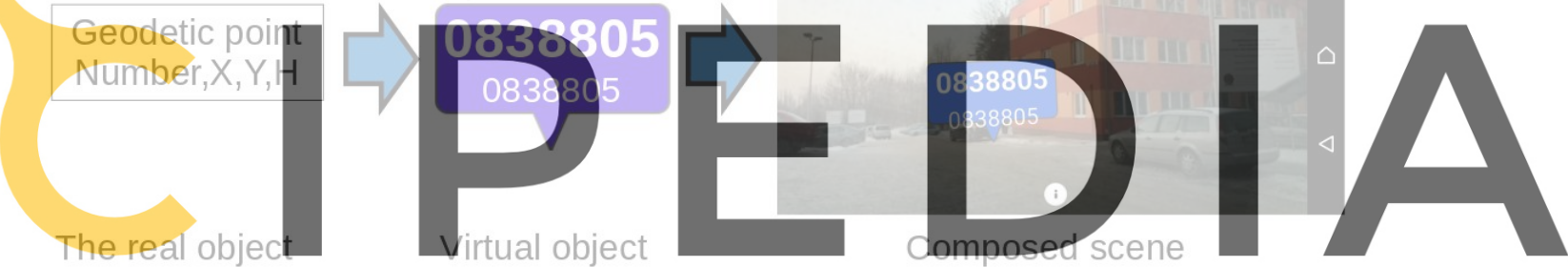

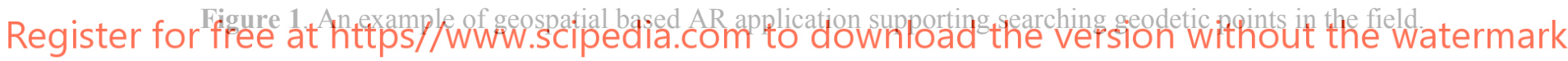

To compose a scene in geospatial based AR, algorithm needs the user's location and orientation in a geographic coordinate space. Determination of location is based on geopositioning techniques such as GPS/GNSS, WiFi or other (e.g. Bluetooth). Sometimes, location can be determined by scanning specially prepared marker for that purpose. Device's orientation parameters like rotation, slope or direction are approximated, using sensors such as a digital compass, accelerometer or gyroscope. Once approximated device's orientation may be also refined by using computer vision techniques. The virtual object can be any digital object like image or icon. To place the virtual object in the right place on the screen and compose the AR scene, the real feature's location is also needed. The augmentation occurs when user is in the vicinity of real object and points on it with a device's camera. The software will automatically determine the location and orientation of the device and display respectively generated image (virtual object) on the screen (Lechner 2015). Figure 1 demonstrates the example of augmentation performed in described way. In this case there's a database of geodetic points, which are placed in the field. AR application connects to database of points and selects these, which are in the vicinity of the device. Then the augmentation process occurs and the searched point appears on the screen (Bednarczyk 2017(b)). 
In computer-vision based AR, the computer vision algorithms are used to process the image viewed by the camera, and compare it with the reference images. Augmentation is obtained when application recognizes and identifies fragments of an image and displays on this place virtual objects. Figure 2 shows the example of augmentation performed this way. In this example, the reference images are created from raster image fragments of analog map. They represent each building on the map. Each building has it's individual virtual representation in a form of automatically generated icon with basic information acquired from GIS database. Furthermore each virtual icon has an interactive button "Details". When user directs the smartphone camera to the map, application recognizes the reference image and displays a virtual icon at this place. When user touches the "Details" button on the screen, application leeds him to additional information selected from database and displayed in a form of website (Bednarczyk 2017(a)).

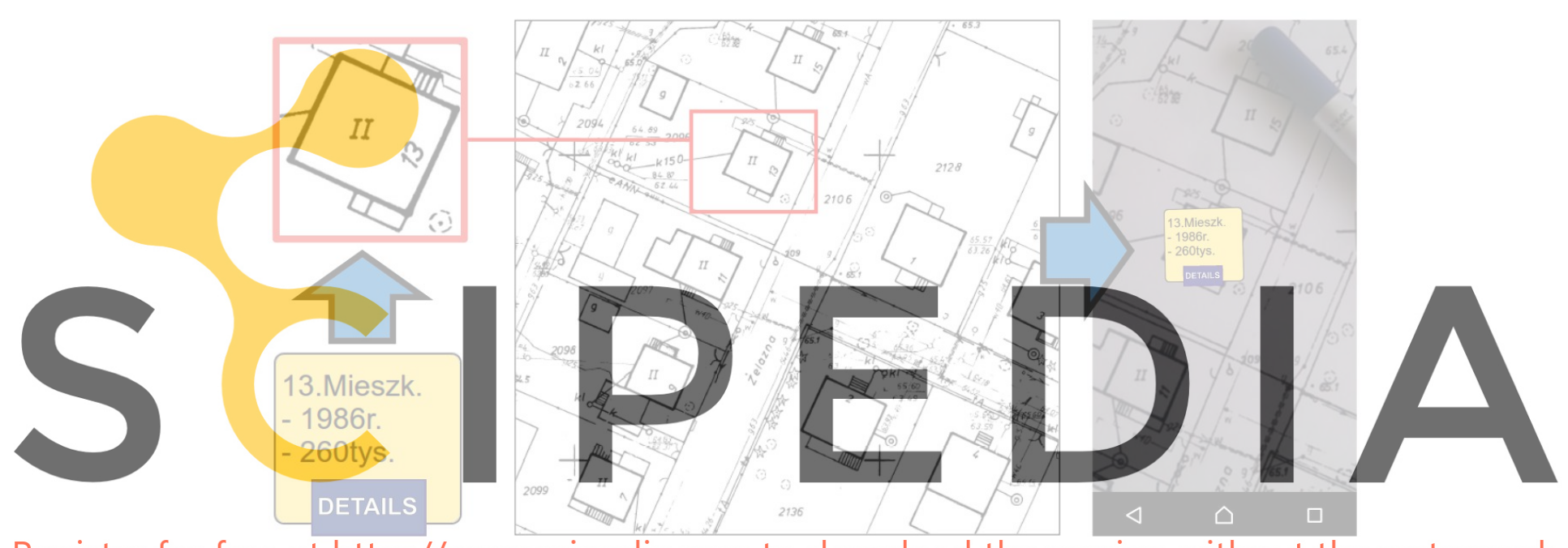

Register for free at https//www.scipedia.com to download the version without the watermark Figure 2. An example of computer-vision based AR on analog map.

\section{Building Facade Visualization}

As mentioned earlier, AR technology can be helpful in visualizing information about the state of the building facade during inspection. By mixing the real view of the object with the previously collected data, a direct comparison of the current and historical status can be obtained. A working prototype of the application, shown in fig. 3 uses computer-vision based AR to recognize the facade of a specific building. After that, an image (drawing) is overlayed that can contain any previously collected information related to the building, such as:

- descriptions and markings of construction elements

- descriptions and markings of defects and damages

- installation descriptions and markings

- interactive elements - to get additional information or perform an action in the application

- historical facade photos

- other useful information 

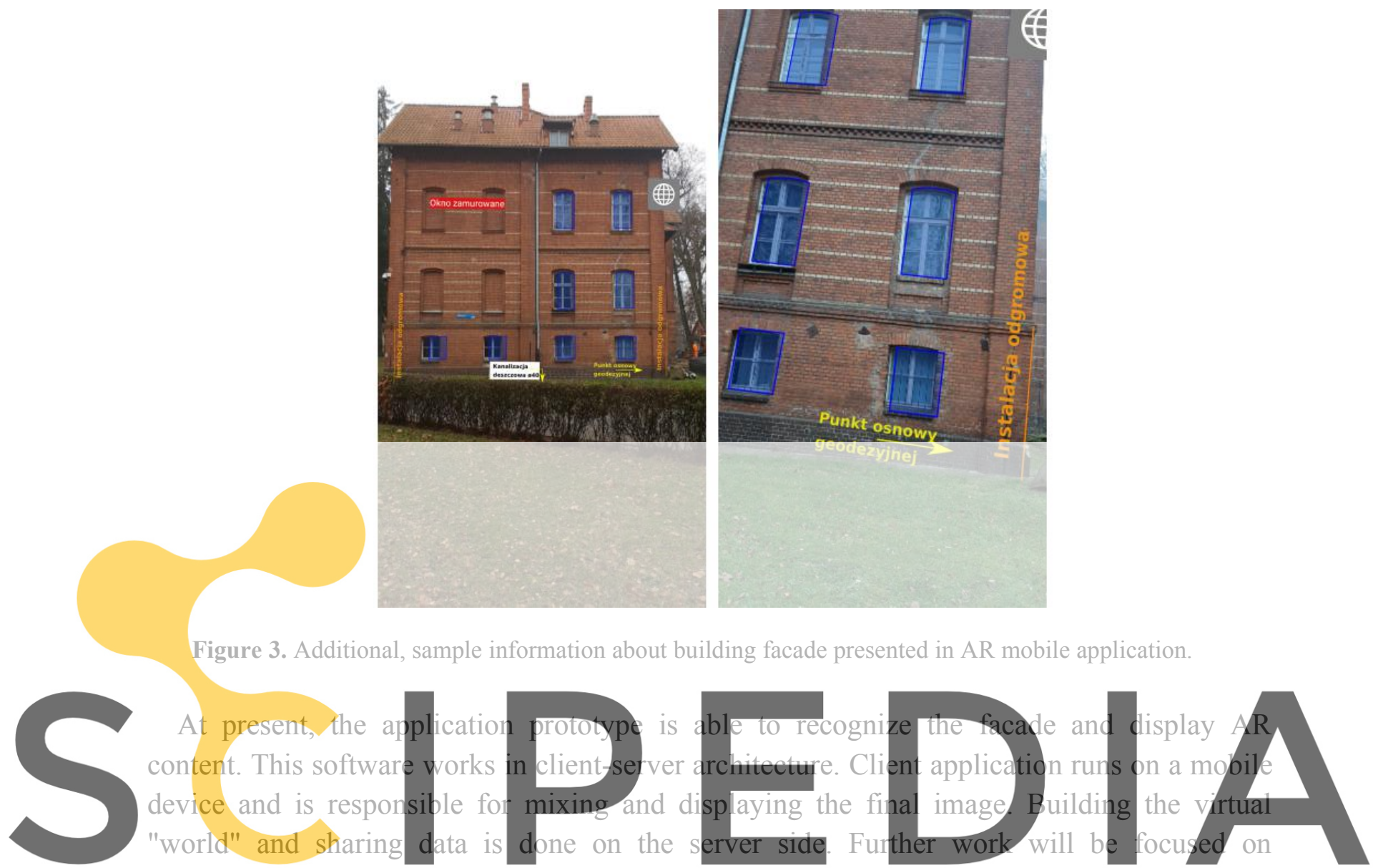

expanding the system's functionality.

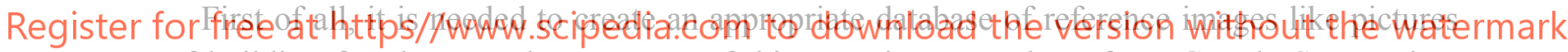

of building facades. For the purposes of this experiment, a photo from Google Street View

was used. It seems that in many cases this may be a sufficient source of images. However, this is a collection obtained automatically and, as a result, there is a risk that there may be photos taken at the wrong angle or some buildings may have been obstructed by obstacles (vegetation, people, vehicles, etc.). In addition, Street View vehicles do not visit every street or passage, but only the most important ones. Still, as of today, Street View appears to be the largest source of data of its kind.

Another important component is the inspections database of individual buildings. For testing purposes, the virtual image has been prepared manually in PNG format. It should be possible to save this data partially in vector format in a database. Thanks to this, it will be possible to generate virtual objects automatically.

To optimize performance and limit data transfer, the application should include an algorithm that allows selection of only those inspections that are closest to the observer. This can be solved by performing database queries taking into account GPS / GNSS location of the mobile device. If the proposed software is to be useful, in addition to viewing inspection data, it should be possible to edit it on site in real time. Thanks to this, inspection data could be supplemented based on the existing technical condition of the facade. All of proposed software should work under control, or be part of, more general system such as GIS or other 
spatial information processing system of this kind. Schema of the conception of proposed AR system for building facades is presented in fig. 4 .

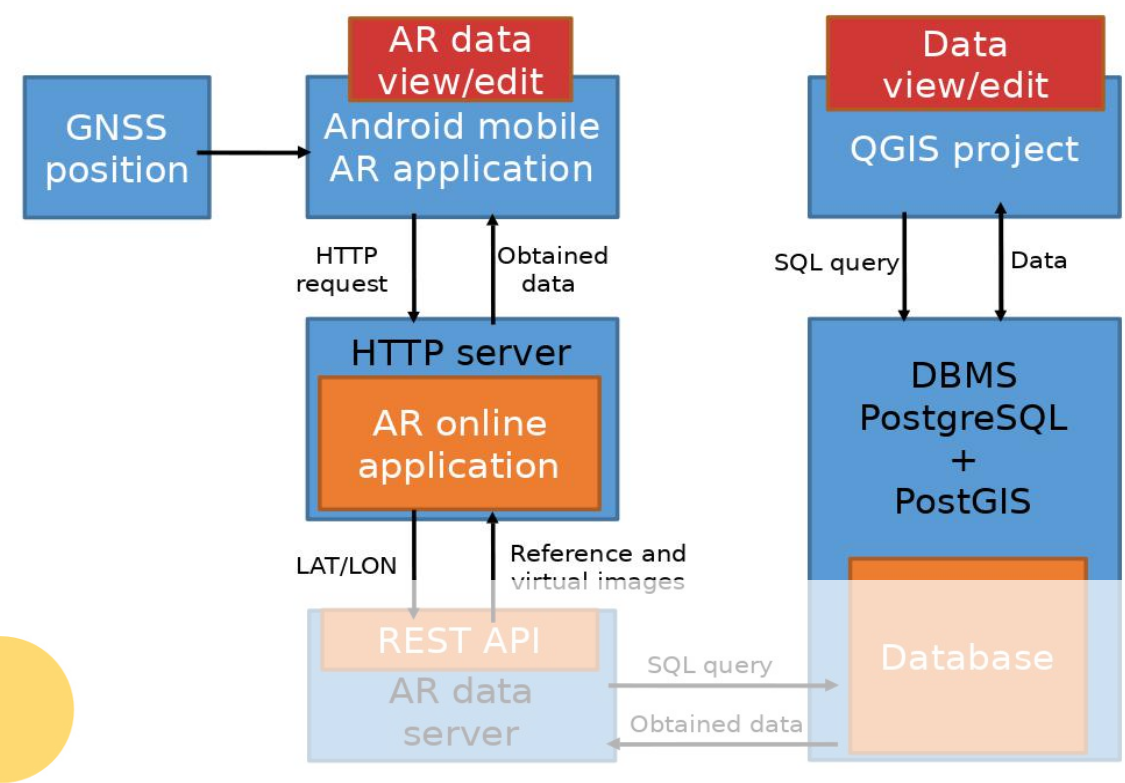

Figure 4. Components of proposed AR system for building facades.

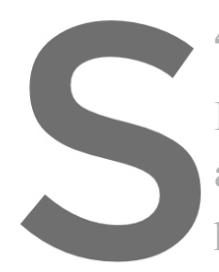

\section{Additional Observations and Resullts}

In author's previous work on AR, some disa

augmented reality scene (Bednarczyk 2017 problem is accuracy

objects location displayed on the smartphone screen. If the GPS position error is small, AR
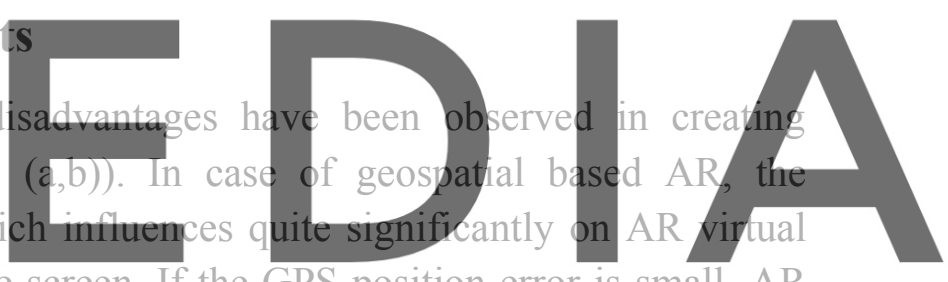

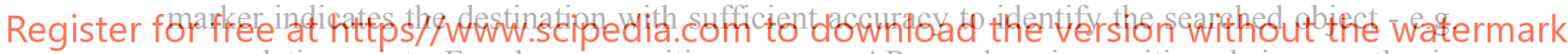

a geodetic point. For larger position errors, AR marker is positioned incorrectly in

inappropriate place. Sometimes there is also a complete lack of GPS signal (e.g. in the forest, urban canion or while cloudy day), then the application does not work properly. This can be remedied by cutting off these observations that give too little accuracy in position determination. However, minor errors, if they occur frequently, will cause the effect of "swimming" or "jumping" virtual markers on the screen. In case of computer vision based AR, no such effects were found, but only point-oriented markers on small fragments of the analog map observed from a short distance - a few centimeters - were examined. In addition, their appearance was compact in form of an simple icon.

In this article, the virtual image covered larger area, the drawing was composed of many elements, and observation distance was several meters. In this case, it was observed that the position of the virtual image begins to deviate from the originally assumed, when changing view perspective or distance. Therefore, additional correction algorithms should be used to improve the generation of the AR scene in such cases, which will be considered in future work. 


\section{Conclusions}

It can be assumed that visualization of building facades using AR can support the process of collecting data in the field. Using AR technology, one can display data from previous inspections, BIM, a database, or e.g. archival photos, overlaying them on the actual image of the building in real time in the field. Thanks to this, one can easily make a comparison with the existing state, make a quick view, take notes and decide whether to update the data.

However, in order to ultimately achieve the goal, further work needs to be done to refine the proposed solution. This applies above all to the system architecture, which should be expanded with appropriate components to ensure efficient and effective work, such as database of inspections and reference images. In addition, it is worth considering refining the algorithm responsible for generating the AR scene in case when observer changes the view perspective or approaches facade of the building.

Finally, considering the pros and cons of two different methods of generating an AR scene, it can be stated that, in case of facades visualization, computer vision based AR gives better results when creating an AR scene than geospatial based AR.

ORCIID

Michał Bednarczyk: https://orcid.org/0000-0002-0450-5327

Tomasz Templin: https://orcid.org/0000-0003-2188-0097
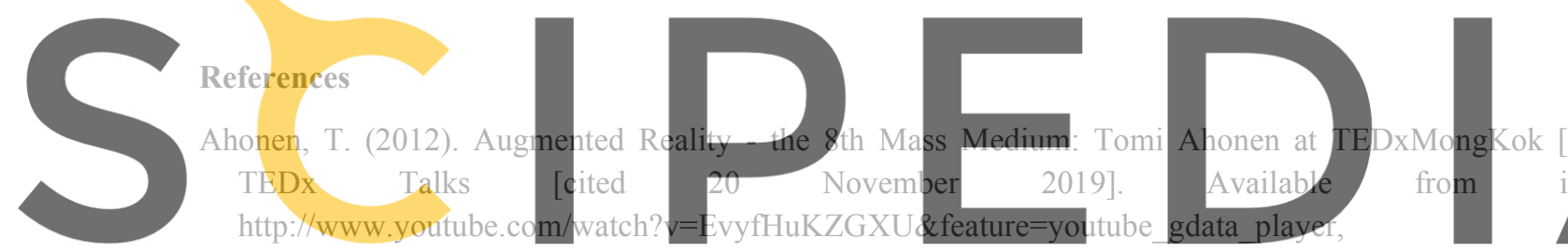

AR Community. (2017). AR Glossary [online]. AR Community for Open and Interoperable Augmented Reality

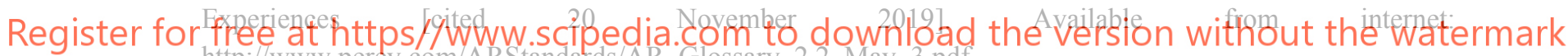
http://www.perey.com/ARStandards/AR_Glossary_2.2_May_3.pdf

Azuma, R. (1997). A Survey of Augmented Reality. Presence: Teleoperators and Virtual Environments 6, no. 4 (1997): 355-385.

Bednarczyk M.(a), APPLICATION POSSIBILITIES OF AUGMENTED REALITY IN ANALOG MAPS, Geographic Information Systems Conference and Exhibition "GIS ODYSSEY 2017" Conference Proceedings, Italy 4th to 8th of September 2017, Trento, Vattaro, ISSN 2459-7627, http://gis.us.edu.pl/index.php/past-gis-conferences/24-gis-odyssey-2017

Bednarczyk M.(b), THE USE OF AUGMENTED REALITY IN GEOMATICS, 10th International Conference "Environmental Engineering", Vilnius, Lithuania, 27-28 April 2017, DOI: https://doi.org/10.3846/enviro.2017.162

Broschart, D. Zeile P. Streich B. (2013). Augmented Reality as a Communication Tool in Urban Design Processes, in REAL CORP 2013 Tagungsband Conference, 20-23 May 2013, Rome, Italy.

Gibert V., Serrat C. and Casas J.R. (2014). Determination of criteria for the exploration and for obtaining indicators in evolutional analysis of degradation in urban facades, in Proceeding of the 13th International Conference on Durability of Building Materials and Components, Sao Paulo, Brasil, 656-63.

Henrysson, A. (2007). Bringing Augmented Reality to Mobile Phones: Doctoral dissertation. Department of Science and Technology Linköpings Universitet, Norrköping.

Höllerer, T.; Feiner, S. (2004). Mobile Augmented Reality, Chapter 9 in Karimi, H.; Hammad, A. (Eds.). Telegeoinformatics: Location-Based, Computing and Services. Taylor and Francis Books Ltd., London, UK.

Janowski A., Bednarczyk M., (2016). Considerations on Indoor Navigation Based on Cheap Mobile Devices, 2016 Baltic Geodetic Congress (BGC Geomatics), At Gdańsk, Poland, DOI: 10.1109/BGC.Geomatics.2016.23 
Kounavis, C. D. Kasimati, A. E.; Zamani, E. D. (2012). Enhancing the Tourism Experience through Mobile Augmented Reality: Challenges and Prospects. International Journal of Engineering Business Management, vol.4.

Lechner, M. (Ed.). (2015). OGC Augmented Reality Markup Language 2.0 (ARML 2.0). Open Geospatial Consortium.

Moloney, J.; Bharat, D. (2011). From Abstraction to Being There: Mixed Reality at the Early Stages of Design. International Journal of Architectural Computing 9, no. 1 (2011): 1-16.

Serrat C. and Gibert V. (2011). Survival analysis methodology for service live prediction and building maintenance, in Proceeding of the 12th International Conference on Durability of Building Materials and Components, vol. II, Porto, Portugal, 599-606.

Serrat C., Gibert V., Rapinski J., and Casas J.R., Followup and Decision (FAD) as a Strategic Tool for Building Stock Management, in Proceedings of the 2016 People, Buildings and Environment Conference, Luhacovice, Czech Republic, 2016.

Siltanen, S. (2012). Theory and applications of marker-based augmented reality. VTT Technical Research Centre of Finland.

Xiangyu, W. (2009). Augmented Reality in Architecture and Design: Potentials and Challenges for Application. International Journal of Architectural Computing, iss. 02, vol. 07, p.309-326.
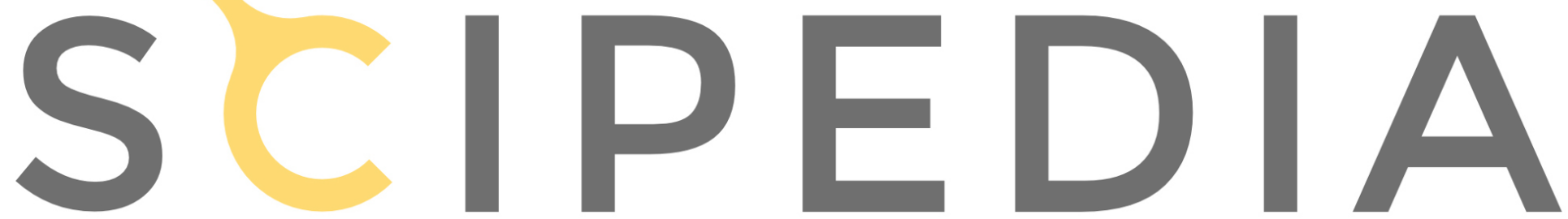